\title{
TWO-PERSON GAME WITH HESITANT FUZZY PAYOFF: AN APPLICATION IN MADM
}

\author{
Jishu JANA AND SANKAR KUMAR ROY*(1)
}

\begin{abstract}
Hesitant Fuzzy Set (HFS) permits the membership function having a collection of probable values which are more effective for modelling the real-life problems. Multiple Attribute Decision Making (MADM) process apparently assesses multiple conflicting attribute in decision making. In traditional decision making problems, each player is moving independently whereas in reality it is seen that each player aims to maximize personal profit which causes a negative impact on other player. MADM problem treats with candidate to the best alternative corresponding to the several attributes. Here, we present an MADM problem under hesitant fuzzy information and then transforming it into two-person matrix game, referred to herein as MADM game. The Technique for Order Preference by Similarity to Ideal Solution (TOPSIS) is one of the prominent approach for solving the MADM problems. In this work, we develop the TOPSIS based on Ordered Weighted Aggregation (OWA) operator and hybrid hesitant fuzzy normalized Euclidean distance. Then the two approaches, namely Hybrid Hesitant Fuzzy Ordered Weighted Aggregation-TOPSIS (HHFOWA-TOPSIS) and the Linear Programming Problem (LPP) are applied to solve the formulated MADM game. For solving MADM game, we apply LPP by considering the various values of $\alpha, \psi$, and HHFOWA-TOPSIS for finding the optimal alternative according to their scores. An investment selection problem is included to explain the feasibility and superiority of our formulated approaches. A comparison analysis is drawn among the obtained results which are derived from the two approaches. LPP and HHFOWA-TOPSIS provide the best alternative for the proposed problem. Finally, conclusions about our findings and outlooks are described.
\end{abstract}

Mathematics Subject Classification. 91A05, 91A35, 03E72, 06D72, $62 \mathrm{C} 86$.

Received August 9, 2020. Accepted September 20, 2021.

\section{INTRODUCTION}

Game theory is predominantly employed as a skilful task for decision making problems in the competitive scheme. In this regard how to settle decisions in competitive situation is as common and important one. The competitive situations are arisen on business model, economics, political science, management science, computer science and engineering etc. Game theory constructs with how Decision Makers (DMs) make judgement when they defend with precise payoffs. For this reason, the knowledge of game theory is graceful in business environment and industry. In competitive systems, matrix game has numerous significant appliances, for example,

Keywords. Matrix games, multiple attribute decision making, hesitant fuzzy set, aggregation operator, HHFOWA-TOPSIS.

Department of Applied Mathematics with Oceanology and Computer Programming, Vidyasagar University, Midnapore 721102, West Bengal, India.

*Corresponding author: sankroy2006@gmail.com

(c) The authors. Published by EDP Sciences, ROADEF, SMAI 2021

This is an Open Access article distributed under the terms of the Creative Commons Attribution License (https://creativecommons.org/licenses/by/4.0), which permits unrestricted use, distribution, and reproduction in any medium, provided the original work is properly cited. 
investment policy, military battles, sport competitions, and various types of competition. In the real-life problem, because of absence of available data, the payoffs of the matrix games are not in precise form. The DMs are behaved here as the players. A two-person zero-sum game is the normal game displayed by including two players, where the matrix games are considered with crisp payoffs. At that point, one player wins and whereby other player losses when two players pick the pure strategies $S_{1}=\left\{\alpha_{i}: i=1,2, \ldots, m\right\}$ and $S_{2}=\left\{\beta_{j}: j=1,2, \ldots, n\right\}$ respectively.

In conventional MADM process, it is assumed that the determination of alternatives corresponding to attributes are exactly known by DM. But in real-life situations, DM does not know exactly the determination of alternatives corresponding to the attributes in uncertain environment. The uncertainty in determinations may be of various types such as probabilistic, fuzzy, fuzzy-probabilistic etc. Here we deal with uncertainty of hesitant fuzzy (Torra and Narukawa [49]) type which is the generalized version of fuzzy set (Zadeh [55]). Here DM chooses a hesitant fuzzy MADM problem. The most useful tool to tackle such type of problems is the hesitant fuzzy set.

Numerous approaches have been discussed for analyzing fuzzy MADM problems by many researchers. We can divide them into two parts. First one is the methods that apply in different ways of ranking fuzzy numbers. So the score of each alternative is computed. Then the best alternative is selected by the score function. The second one depends on various ordering of fuzzy numbers. In traditional MADM methods, TOPSIS has been paid great attention by researchers and scientists. Originally, the concept of TOPSIS was introduced by Hwang and Yoon [15]. The basic theory of TOPSIS is that the selected alternative should have the shortest distance from the positive ideal solution (PIS) and largest distance from the negative ideal solution (NIS). Due to its flexibility and comprehensibility, it is widely applied in various fields. Yoon and Kim [54] analyzed the behavioral TOPSIS in decision making. Hatami-Marbini and Kangi [14] proposed an extension of fuzzy TOPSIS for a group decision making with an application to Tehran stock exchange. Wang and Chen [50] studied an MADM based on intervalvalued intuitionistic fuzzy sets and solved it by simplex algorithm and extended TOPSIS. Liang and Xu [27] worked out a new extension of TOPSIS method for Multiple Criteria Decision Making (MCDM) with hesitant Pythagorean fuzzy sets. Aggarwal [2] elaborated hesitant fuzzy information sets and its application in group decision making. Lalotra and Singh [24] described knowledge measure of hesitant fuzzy set and its application in multi-attribute decision-making. Xiong et al. [52] presented a novel MAGDM approach with proportional hesitant fuzzy sets. Xia and Xu [51] proposed hesitant fuzzy information aggregation in decision making. Liao and $\mathrm{Xu}[28]$ depicted some new hybrid weighted aggregation operators under hesitant fuzzy MCDM environment. Jana and Roy [18] worked on soft matrix game by using hesitant fuzzy MCDM approach. Jin et al. [19] offered ordered weighted averaging aggregation on convex poset. Also, Sun et al. [48] considered an innovative TOPSIS approach based on hesitant fuzzy correlation coefficient and its applications. Chen and Hwang [10] unfolded the most appropriate method for solving MADM problem under fuzzy environment. Farhadinia and Xu [13] presented a novel distance-based multiple attribute decision-making with hesitant fuzzy sets. Mo et al. [34] unfolded feature-based hesitant fuzzy aggregation method for satisfaction with life scale. Bhaumik and Roy [5] proposed different aggregation operators in intuitionistic interval-valued hesitant fuzzy matrix games for solving management problems. Mula et al. [35] analyzed bi-matrix games with birough payoff elements and solved with the help of birough programming approach. Chen and Larbani [11] described a new method for analyzing a fuzzy MADM problem by reducing it into a two-person zero-sum game by using $\alpha$-cut. Also, Roy [38] elaborated multi-criteria decision making and fuzzy set theory in game environment. Jana and Roy [16] studied solution of matrix games with generalized trapezoidal fuzzy payoffs. Campos [9] offered fuzzy linear programming models to solve fuzzy matrix games. Also, Bhaumik et al. [6] presented robust ranking technique for analyzing a triangular intuitionistic fuzzy matrix game. Roy and Mondal [41] worked out an approach for solving fuzzy interval-valued matrix game. Bector et al. [4] offered duality in linear programming with fuzzy parameters and matrix games with fuzzy payoffs. Roy and Bhaumik [43] unfolded intelligent water management problem with the help of triangular type-2 intuitionistic fuzzy two-person zero-sum games approach. Larbani [25] analyzed bimatrix game with fuzzy payoffs by introducing nature as third player. Roy and Mula ([39, 40]) proposed rough set approach in rough bimatrix game environment. Aggarwal and Khan [3] elaborated 
TABLE 1. Notation list include sets, parameters and decision variables.

\begin{tabular}{|c|c|c|}
\hline Sets & Parameters & Decision variables \\
\hline$\tilde{\tilde{A}}$ : Hesitant fuzzy sets & $w$ : Weight of each alternative & $\begin{array}{l}X \text { i.e., } X=\left\{x: \sum_{i=1}^{m} x_{i}=\right. \\
\left.1, x_{i} \geq 0, i=1,2, \ldots, m\right\}\end{array}$ \\
\hline$h_{\tilde{\tilde{A}}}(x):$ Hesitant fuzzy element & $\lambda: \lambda \in[0,1]$ & $\begin{array}{l}Y=\left\{y: \sum_{j=1}^{n} y_{j}=1, y_{j} \geq\right. \\
0, j=1,2, \ldots, n\}\end{array}$ \\
\hline $\begin{array}{l}\tilde{\tilde{h}}_{j} \text { and } \tilde{\tilde{h}}_{j}: \text { Distinct collection } \\
\text { of hesitant fuzzy sets }\end{array}$ & $\bar{\lambda}:[0,1]$ & $x_{\alpha}^{\prime *}:$ Optimal solution \\
\hline $\begin{array}{l}\tilde{\tilde{a}} \text { : Triangular hesitant fuzzy } \\
\text { number }\end{array}$ & $\begin{array}{l}\psi: \psi \in[0,1] \text { which describes } \\
\text { the significance pointed to the } \\
\text { lower expected value }\end{array}$ & $y_{\alpha}^{\prime *}$ : Optimal solution \\
\hline $\begin{array}{l}\tilde{\tilde{A}}=\left[\tilde{\tilde{a}}_{i j}\right]_{m \times n}= \\
\left(A^{L}, A^{C}, A^{U}\right): \text { Triangular } \\
\text { hesitant fuzzy matrix }\end{array}$ & $\begin{array}{l}(1-\psi): \text { Indicate the signif- } \\
\text { icance pointed to the upper } \\
\text { expected value }\end{array}$ & \\
\hline $\begin{array}{l}\tilde{\tilde{A}}_{\alpha}: \alpha \text {-cut of triangular hesi- } \\
\text { tant fuzzy matrix }\end{array}$ & & \\
\hline
\end{tabular}

multi-objective fuzzy matrix games via multi-objective linear programming approach. Bigdeli and Hassanpour [8] discussed an approach to solve multi-objective linear production planning games with fuzzy parameters. Roy and Mula [42] analyzed matrix game with rough payoffs using genetic algorithm. Moreover, Larbani [26] viewed on non-coopertaive fuzzy games in normal form. Jana and Roy [17] discussed about dual hesitant fuzzy matrix games based on new similarity measure. Bhaumik et al. [7] studied Prisoners' dilemma game theory by using TOPSIS with the help of hesitant interval-valued intuitionistic fuzzy-linguistic term set approach. Ezimadu [12] viewed cooperative advertising decisions in a manufacturer-distributor-retailer supply chain by using game theory. Singh and Lalotra [47] presented generalized correlation coefficients of the hesitant fuzzy sets with their application to clustering analysis. Achemine et al. [1] analyzed $z$-equilibria in bi-matrix games with uncertain payoffs. More recently, Roy and Jana [44] discussed on multi-objective linear production linear production planning game in triangular hesitant fuzzy set. Recently, Khalilpourazari and Doulabi [20] designed a hybrid reinforcement learning based algorithm with application in prediction of the COVID-19 pandemic in Quebec. Khalilpourazari et al. [23] proposed gradient-based grey wolf optimizer with Gaussian walk and its application in modelling and prediction of the COVID-19 pandemic. Khalilpourazari and Doulabi [21] analyzed robust modelling and prediction of the COVID-19 pandemic in Canada. Khalilpourazari and Pasandideh [22] designed emergency flood evacuation plans using robust optimization and artificial intelligence. From the recent literature survey, we notice that a lot of papers has been discussed on MADM game under fuzzy environment. But to the best of our knowledge, till now no one did consider the MADM game with hesitant fuzzy environment. A complete notation list include sets, parameters and decision variables is shown in Table 1.

However, there are various distances, similarity and correlation coefficient measure for HFS and its extension has been developed to make the decision with the help of TOPSIS. So it is essential to construct new approach to tackle this situation. The goal of this study is to develop an MADM game under hesitant fuzzy environment and to find the best alternative for the real-life application. Inspired by the above research works, we propose an HHFOWA-TOPSIS to solve the hesitant fuzzy MADM game. In this game the two players are denoted as actual DM and Nature. So here DM is the player I and Nature is the player II. Player I attempts to maximize the minimum payoffs whereas player II efforts to minimize the maximum lose. An extensive comparison of different characteristics between the present study and related works in these direction is shown in Table 2. The major contributions of this paper are summarized in three aspects, which are as follows: 
TABLE 2. Comparisons of different authors' contributions.

\begin{tabular}{lccccc}
\hline \hline Author(s) & LPP & $\alpha$-cut & Hesitancy & TOPSIS & Environment \\
\hline $\begin{array}{l}\text { Bector and } \\
\text { Chandra [4] }\end{array}$ & $\checkmark$ & & & & Fuzzy set \\
\hline Campos [9] & $\checkmark$ & & & & Fuzzy set \\
\hline $\begin{array}{l}\text { Chen and } \\
\text { Larbani [11] }\end{array}$ & $\checkmark$ & $\checkmark$ & & & Fuzzy MADM \\
\hline $\begin{array}{l}\text { Liang and Xu } \\
{[27]}\end{array}$ & & & $\checkmark$ & $\checkmark$ & Hesitant fuzzy set \\
\hline $\begin{array}{l}\text { Sakawa and } \\
\text { Yano [45] }\end{array}$ & $\checkmark$ & $\checkmark$ & & & Fuzzy set \\
\hline \begin{tabular}{l} 
This investigation \\
\hline
\end{tabular} & $\checkmark$ & $\checkmark$ & $\checkmark$ & $\checkmark$ & $\begin{array}{l}\text { Hesitant fuzzy } \\
\text { MADM }\end{array}$ \\
\hline
\end{tabular}

- A game theory oriented MADM problem is developed under hesitant fuzzy environment and then it is converted into crisp MADM game by using $\alpha$-cut and maximin principle.

- The idea of LPP is established to obtain optimal strategy of the crisp MADM game and a new score function is introduced to get the ranking order of alternatives.

- The concept of HHFOWA-TOPSIS based on OWA and hybrid hesitant fuzzy normalized Euclidean distance is introduced to solve the hesitant fuzzy MADM game.

The rest of the paper is organized as follows: In Section 2, we keep some basic definitions of two-person zero-sum games, hesitant fuzzy sets, score function, aggregation operator, hybrid hesitant normalized Euclidean distance and membership function. In Section 3, we convert the basic MADM problem with hesitant fuzzy decision matrix into crisp MADM game by $\alpha$-cut and maximin principle. We develop the solution procedure for MADM game by incorporating two algorithms in Section 4. In Section 5, an investment selection problem is considered to check the applicability of LPP and HHFOWA-TOPSIS approach, and the results are discussed. In Section 6, we find a likeness of our outcomes with the similar papers. At last, conclusions are described in Section 7 with the suggestions of future study.

\section{Preliminaries}

Here we include the concept of matrix game, and some basic definitions of HFSs, score function, aggregation operator, hybrid hesitant normalized Euclidean distance and membership function.

\subsection{Concept of two-person zero-sum game}

Game theory is mainly connected with the strategies interaction of DMs and they are known that their strategies depend on one another. A two-person zero-sum game is the normal game which includes two players; and considering that both the players have pure and mixed strategies. In matrix game, non-cooperation is more interesting than cooperation due to realistic situation. In practical situation, the payoffs of cooperation and non-cooperation are in the sense of fuzzy understanding. A two-person zero-sum matrix game can be stated in the following payoff matrix as:

$$
A=\left[\begin{array}{cccc}
a_{11} & a_{12} & \ldots & a_{1 n} \\
a_{21} & a_{22} & \ldots & a_{2 n} \\
\vdots & \vdots & & \vdots \\
a_{m 1} & a_{m 2} & \ldots & a_{m n}
\end{array}\right]
$$


where $A$ is the payoff matrix of player I. All the elements in the matrix game are considered to be positive in this paper. Since this is the two-person zero-sum game so the payoffs of the player II is $-A$.

Definition 2.1. The mixed strategy of player I is described by $m$-tuple $x=\left(x_{1}, x_{2}, \ldots, x_{m}\right)^{t}$ of probabilities that add to 1 i.e., $x^{t} e_{m}=1$, where $e_{m}$ is a vector whose each component is equal to 1 . Similarly we define the mixed strategy of player II.

Definition 2.2. The mixed strategies of players I and II be $x$ and $y$ respectively, then the expected payoff of player I is defined as $x^{t} A y=\sum_{i=1}^{m} \sum_{j=1}^{n} a_{i j} x_{i} y_{j}$. So the expected payoff of player II is $-x^{t} A y$.

Definition 2.3. Let $x^{*}$ and $y^{*}$ be the mixed strategies of players I and II respectively, are said to be optimal strategies if $x^{t} A y^{*} \leq x^{t *} A y^{*}$ and $x^{t *} A y^{*} \leq x^{t *} A y$ for all mixed strategies $x$ and $y$.

Corollary 2.4. (Parthasarathy and Raghavan [37]) Let $x^{*}$ be the solution of optimization problem

$$
\begin{aligned}
& \max _{x}\left\{\min \left\{\sum_{i=1}^{m} a_{i 1} x_{i}, \sum_{i=1}^{m} a_{i 2} x_{i}, \ldots, \sum_{i=1}^{m} a_{i n} x_{i}\right\}\right\} \\
& \text { subject to } x^{t} e_{m}=1 \\
& x_{i} \geq 0, \forall i, e_{m}=(1,1, \ldots, 1)
\end{aligned}
$$

which is an optimal strategy of player I, whereas any solution $y^{*}$ of the optimization problem

$$
\begin{aligned}
& \min _{y}\left\{\max \left\{\sum_{j=1}^{n} a_{1 j} y_{j}, \sum_{j=1}^{n} a_{2 j} y_{j}, \ldots, \sum_{j=1}^{n} a_{n j} y_{j}\right\}\right\} \\
& \text { subject to } y^{t} e_{n}=1 \\
& y_{j} \geq 0, \forall j, e_{n}=(1,1, \ldots, 1)
\end{aligned}
$$

is an optimal strategy of player II.

Corollary 2.5. (Parthasarathy and Raghavan [37]) Considering a payoff matrix A, then on calculating the optimal strategies which are defined in equations (2.2) and (2.3), equivalent to solve (MOD-1) and (MOD-2) as:

$$
\begin{aligned}
& \max _{x}\left\{\min \left\{\sum_{i=1}^{m} a_{i 1} x_{i}, \sum_{i=1}^{m} a_{i 2} x_{i}, \ldots, \sum_{i=1}^{m} a_{i n} x_{i}\right\}\right\} \\
& \text { subject to } x^{t} e_{m}=1 \\
& x_{i} \geq 0, \forall i, e_{m}=(1,1, \ldots, 1),
\end{aligned}
$$

which then an optimal strategy of player I, whereas any solution $y^{*}$ of the optimization problem

$$
\begin{aligned}
& \min _{y}\left\{\max \left\{\sum_{j=1}^{n} a_{1 j} y_{j}, \sum_{j=1}^{n} a_{2 j} y_{j}, \ldots, \sum_{j=1}^{n} a_{n j} y_{j}\right\}\right\} \\
& \text { subject to } y^{t} e_{n}=1 \\
& y_{j} \geq 0, \forall j, e_{n}=(1,1, \ldots, 1),
\end{aligned}
$$

is an optimal strategy of player II. 
Corollary 2.6. ([37]): Assuming a payoff matrix A, calculating optimal strategies defined in equations (2.2) and (2.3) are equivalent to solve (MOD-1) and (MOD-2) as:

$$
\begin{array}{ll}
\left(\text { MOD - 1) } \min _{x} x^{\prime t} e_{m}\right. & \left(\text { MOD - 2) } \max _{y} y^{\prime t} e_{n}\right. \\
\text { subject to }\left\{\begin{array}{l}
x^{\prime}{ }^{t} A \geq e_{n}^{t}, \\
x_{i}^{\prime} \geq 0, \forall i
\end{array}\right. & \text { subject to }\left\{\begin{array}{l}
A y^{\prime} \leq e_{m}, \\
y_{j}^{\prime} \geq 0, \forall j .
\end{array}\right.
\end{array}
$$

Corollary 2.7. Problems (2.2) and (2.3) of players I and II can be rewritten in the following models respectively:

$$
\begin{array}{rr}
(\text { MOD }-\mathbf{3}) \max _{x} v & (\mathbf{M O D}-\mathbf{4}) \min _{y} w \\
\text { subject to } & \left\{\begin{array} { l } 
{ x ^ { t } A \geq v e _ { n } ^ { t } } \\
{ x ^ { t } e _ { m } = 1 } \\
{ x _ { i } \geq 0 , \forall i }
\end{array} \text { subject to } \left\{\begin{array}{l}
A y \leq w e_{m} \\
y^{t} e_{n}=1 \\
y_{j} \geq 0, \forall j .
\end{array}\right.\right.
\end{array}
$$

It is easy to observe that from (MOD-3) and (MOD-4), we get $v=\min _{j}\left\{\sum_{i=1}^{m} a_{i j} x_{i}\right\}$ and $w=$ $\max _{i}\left\{\sum_{j=1}^{n} a_{i j} y_{j}\right\}$ respectively.

From Corollary 2.6, we discuss the subsequent steps for analyzing the game problem. First, we solve (MOD-1) and (MOD-2) and assume that $x^{\prime *}$ and $y^{\prime *}$ be the optimal solutions respectively. Secondly, to receive the optimal strategy $x^{*}$ of player I, first we determine the value $v$ by the formula $\sum_{i=1}^{m} x_{i}^{\prime *}=1 / v$. Then applying the transformation $x^{\prime}=x / v$, we have the optimal strategy $x^{*}=v x^{\prime *}$. Similarly, we have the optimal strategy for player II. So, by Corollary 2.6, the problem of calculating the sets of optimal strategies is converted to solve two LPPs i.e., (MOD-3) and (MOD-4). The two problems will be applied extensively to the rest of the paper.

\subsection{Hesitant fuzzy number and hesitant fuzzy matrix}

Hesitant fuzzy set is an extremely valuable tools to handle the circumstances where there are some problems to calculate the membership function of an element to a set. Here we introduce the definition of a triangular hesitant fuzzy number in the space of matrices.

Definition 2.8. (Torra and Narukawa [49]): Let $X$ be a reference set, then a hesitant fuzzy set $\tilde{\tilde{A}}$ on $X$ is in term of a function that when used to $X$ returns a subset of $[0,1]$, which can be stated as: $\tilde{\tilde{A}}=\left\{<x, h_{\tilde{A}}(x)>: x \in X\right\}$, where $h_{\tilde{\tilde{A}}}(x)$ is a set of values in $[0,1]$, signifying the probable membership degrees of the element $x \in X$ to the set $\tilde{\tilde{A}}$. Also, $h \tilde{\tilde{A}}(x)$ is mentioned as a Hesitant Fuzzy Element (HFE) and denoted by $h$.

Suppose three HFEs are considered by $\tilde{\tilde{h}}, \tilde{\tilde{h}}_{1}$ and $\tilde{\tilde{h}}_{2}$. Torra and Narukawa [49] defined some operations on them, which can be stated as:

$-\tilde{\tilde{h}}^{c}=\cup_{\gamma \in \tilde{h}}\{1-\gamma\}$,

$-\tilde{\tilde{h}}_{1} \cup \tilde{\tilde{h}}_{2}=\cup_{\gamma_{1} \in \tilde{\tilde{h}}_{1}, \gamma_{2} \in \tilde{\tilde{h}}_{2}} \max \left\{\gamma_{1}, \gamma_{2}\right\}$

$-\tilde{\tilde{h}}_{1} \cap \tilde{\tilde{h}}_{2}=\cap_{\gamma_{1} \in \tilde{\tilde{h}}_{1}, \gamma_{2} \in \tilde{\tilde{h}}_{2}} \min \left\{\gamma_{1}, \gamma_{2}\right\}$,

Definition 2.9. Let $\tilde{\tilde{h}}$ be the non-empty hesitant fuzzy set, $S(\tilde{\tilde{h}})=\frac{1}{\# h} \sum_{\gamma \in \tilde{\tilde{h}}} \gamma$ is called the score value of $\tilde{\tilde{h}}$, where $\# \hat{\tilde{\tilde{h}}}$ is the number of the elements in hesitant fuzzy set $\tilde{\tilde{\tilde{h}}}$. For two hesitant fuzzy sets $\tilde{\tilde{h}}_{1}$ and $\tilde{\tilde{h}}_{2}$, if $S\left(\tilde{\tilde{h}}_{1}\right) \geq S\left(\tilde{\tilde{h}}_{2}\right)$, then $\tilde{\tilde{h}}_{1} \geq \tilde{\tilde{h}}_{2}$ and if $S\left(\tilde{\tilde{h}}_{1}\right)=S\left(\tilde{\tilde{h}}_{2}\right)$, then $\tilde{\tilde{h}}_{1}=\tilde{\tilde{h}}_{2}$.

Now some operations on hesitant fuzzy elements $\tilde{\tilde{h}}, \tilde{\tilde{h}}_{1}$ and $\tilde{\tilde{h}}_{2}$ are expressed based on the relationship between HFEs and intuitionistic fuzzy value as: 
$-\tilde{\tilde{h}}^{\lambda}=\cup_{\gamma \in \tilde{\tilde{h}}}\left\{\gamma^{\lambda}\right\}$

$-\lambda \tilde{\tilde{h}}=\cup_{\gamma \in \tilde{\tilde{h}}}\left\{1-(1-\gamma)^{\lambda}\right\}$,

$-\tilde{\tilde{h}}_{1} \oplus \tilde{\tilde{h}}_{2}=\cup_{\gamma_{1} \in \tilde{\tilde{h}}_{1}, \gamma_{2} \in \tilde{\tilde{h}}_{2}}\left\{\gamma_{1}+\gamma_{2}-\gamma_{1} \gamma_{2}\right\}$,

- $\tilde{\tilde{h}}_{1} \otimes \tilde{\tilde{h}}_{2}=\cup_{\gamma_{1} \in \tilde{\tilde{h}}_{1}, \gamma_{2} \in \tilde{\tilde{h}}_{2}}\left\{\gamma_{1} \gamma_{2}\right\}$.

Distance and similarity measures are most important in the applications of MADM. By using distance measure, we reduce the MADM game with hesitant fuzzy payoffs to crisp payoffs.

Definition 2.10. Let $\tilde{\tilde{h}}_{1}$ and $\tilde{\tilde{h}}_{2}$ be two hesitant fuzzy elements, then the hybrid hesitant normalized Euclidean distance measure within $\tilde{\tilde{h}}_{1}$ and $\tilde{\tilde{h}}_{2}$ is stated as

$$
d\left(\tilde{\tilde{h}}_{1}, \tilde{\tilde{h}}_{2}\right)=\sqrt{\left[\frac{1}{2}\left(\frac{1}{k(\tilde{\tilde{h}})} \sum_{l=1}^{k(\tilde{\tilde{h}})}\left|h_{1}^{\sigma(l)}-h_{2}^{\sigma(l)}\right|^{2}+\max _{j}\left|h_{1}^{\sigma(l)}-h_{2}^{\sigma(l)}\right|^{2}\right)\right]},
$$

where $k(\tilde{\tilde{h}})$ is the number of the elements in $\tilde{\tilde{h}}$, and $h_{1}^{\sigma(l)}$ and $h_{2}^{\sigma(l)}$ are the largest values in $\tilde{\tilde{h}}_{1}$ and $\tilde{\tilde{h}}_{2}$ respectively. In most of the cases, $k\left(\tilde{\tilde{h}}_{1}\right) \neq k\left(\tilde{\tilde{h}}_{2}\right)$ thus, assume that $k=\max \left\{k\left(\tilde{\tilde{h}}_{1}\right), k\left(\tilde{\tilde{h}}_{2}\right)\right\}$. Hence it is essential to extend the smaller one until both of them have similar length when we compare between them. To expand the smaller one, the suitable path is to add the same value many times in it. So the choice of this value basically depends on the DM risk preferences. An optimist bears graceful outcome and may add the maximum value, whereas a pessimist looks for unfavourable results and may attach the minimum value. As for example, let $\tilde{\tilde{h}}_{1}=\{0.3,0.4\}$, $\tilde{\tilde{h}}_{2}=\{0.6,0.7,0.8\}$ and $k\left(\tilde{\tilde{h}}_{1}\right)<k\left(\tilde{\tilde{h}}_{2}\right)$. In this case, the optimist may expand $\tilde{\tilde{h}}_{1}$ to $\tilde{\tilde{h}}_{1}=\{0.3,0.4,0.4\}$ and the pessimist may expand it as $\tilde{\tilde{h}}_{1}=\{0.3,0.3,0.4\}$. Though the result may not be same if we expand the smaller one by adding any value in it. In this paper, we extend the smaller one by adding any value in pessimist way because the DM risk preferences can properly dominance the final decision.

Now the concept of MADM game is defined as follows:

MADM game: The development scenario for game theory is to integrate MADM for tackling the decision making problems. Game theory plays a significant role in various collective negotiations among the various participants. As for example, various negotiations take place when a company (DM) wants to invest lump-sum amount of money but the collective risks are taken together. Then by using game theory, the DM and Nature (collective risks) can arrive at the optimal solution of this issue. So the best path is to combine an MADM with game to establish a decision making model.

Aggregation operator: OWA operator was introduced by Yager [53]. It is a well-known aggregation method, which has been extended and studied by many researchers. Merigo [33] introduced a model between the weighted average and induced OWA operator. Inspired by the idea of OWA operator based on the existing hesitant fuzzy information aggregation and distance measure, we propose a new hesitant fuzzy distance measure, called Hybrid Hesitant Fuzzy Ordered Weighted Average (HHFOWA) operator. It can be defined as follows:

Definition 2.11. Suppose $\tilde{\tilde{h}}_{j}$ and $\tilde{\tilde{h}}_{j}^{\prime}(j=1,2, \ldots, n)$ be two distinct collections of HFSs, and let HHFOWA: $\Omega^{n} \times \Omega^{n} \rightarrow \mathbb{R}$ that has an associated weight vector $W$ with $w_{j} \in[0,1]$ and $\sum_{j=1}^{n} w_{j}=1$, according to the following formula:

$$
\operatorname{HHFOWA}\left(\left(\tilde{\tilde{h}}_{1}, \tilde{\tilde{h}}_{1}^{\prime}\right) \ldots\left(\tilde{\tilde{h}}_{n}, \tilde{\tilde{h}}_{n}^{\prime}\right)\right)=\sum_{j=1}^{n} w_{j} d\left(\tilde{\tilde{h}}_{j}, \tilde{\tilde{h}}_{j}^{\prime}\right) \text {. }
$$


Membership function: Let $\mu_{\tilde{a}}(x)$ be the triangular membership function of hesitant fuzzy number $\tilde{\tilde{a}}$, is defined by $\tilde{\tilde{a}}=\left(a^{L}, a^{C}, a^{U}\right)$ where $a^{L}, a^{C}$ and $a^{U}$ are denoted as lower value, center value and upper value of $\tilde{\tilde{a}}$. Presently we define a hesitant fuzzy decision matrix by expanding the definition of triangular hesitant fuzzy number with membership function. The membership function of the triangular hesitant fuzzy number $\tilde{\tilde{a}}=\left(a^{L}, a^{C}, a^{U}\right)$ is stated on the real line which is described as the set: $L=\left\{a: \exists \lambda \in \mathbb{R}: a=\lambda a^{U}+w(1-\lambda) a^{L}\right\}$. Likewise, membership function for a triangular hesitant fuzzy matrix, we state the real line in the space of the matrices as: $L_{1}=\left\{A: \exists \lambda \in \mathbb{R}: A=\lambda A^{U}+w(1-\lambda) A^{L}\right\}$ where $A^{L}=\left[a_{i j}^{L}\right]_{m \times n}$ and $A^{U}=\left[a_{i j}^{U}\right]_{m \times n}$ are lower and upper bound matrices respectively, with positive real entry such that $a_{i j}^{L}<a_{i j}^{U}$ for all $i$ and $j$. So the line $L_{1}$ is produced by the matrices $A^{L}$ and $A^{U}$. Assume that $A^{C}=\left[a_{i j}^{C}\right]_{m \times n}$ be a matrix and $A^{C} \in\left[A^{L}, A^{U}\right]=\left\{A: A=\lambda A^{L}+w(1-\lambda) A^{U}, \lambda \in[0,1]\right\}$, as in the sense of triangular hesitant fuzzy numbers. So, $A^{C}$ describes the key value of the hesitant fuzzy matrix. From the definition of matrix $A^{C}$, it is obvious that there exists a number $\bar{\lambda} \in[0,1]$ such that $A^{C}=\bar{\lambda} A^{L}+w(1-\bar{\lambda}) A^{U}$.

Definition 2.12. Based on the real line $L_{1}$ and the matrices $A^{L}, A^{C}, A^{U}$ as stated above, we define the membership function of the triangular hesitant fuzzy matrix $\tilde{\tilde{A}}=\left[\tilde{\tilde{a}}_{i j}\right]_{m \times n}=\left(A^{L}, A^{C}, A^{U}\right)$ as follows:

$$
\mu_{\tilde{\tilde{A}}}\left(\lambda A^{U}+w(1-\lambda) A^{L}\right)=\mu(\lambda)=\mu_{\tilde{\tilde{A}}}(A)= \begin{cases}0, & \text { if } \lambda \leq 0, \\ \lambda / \bar{\lambda}, & \text { if } 0<\lambda<\bar{\lambda}, \\ 1, & \text { if } \lambda=\bar{\lambda} \\ w(1-\lambda) /(1-\bar{\lambda}), & \text { if } \bar{\lambda}<\lambda<1 \\ 0, & \text { if } \lambda \geq 1\end{cases}
$$

This is an important to remark that the membership function $\mu(\lambda)=\mu_{\tilde{\tilde{A}}}\left(\lambda A^{U}+w(1-\lambda) A^{L}\right)$ is just the membership function of triangular hesitant fuzzy number $\tilde{\tilde{a}}=(0, \bar{\lambda}, 1)$. So we define the membership function of the hesitant fuzzy matrix $\tilde{\tilde{A}}=\left[\tilde{\tilde{a}}_{i j}\right]_{m \times n}=\left(A^{L}, A^{C}, A^{U}\right)$ with the membership of the hesitant fuzzy number $(0, \bar{\lambda}, 1)$.

Corollary 2.13. It is important to note that in Definition 2.12, we do not consider that each entry $\tilde{\tilde{a}}_{i j}$ of the hesitant fuzzy matrix $\tilde{\tilde{A}}$ as a triangular hesitant fuzzy number i.e., $\tilde{\tilde{a}}_{i j}=\left(a_{i j}^{L}\right.$, $\left.a_{i j}^{C} a_{i j}^{U}\right)$. So in this case $A^{C}=\left[a_{i j}^{C}\right]_{m \times n}$, herein no surety that the number $\lambda$ is applied for considering the matrix $A^{C}$, is free from $i$ and $j$. In our proposed approach, the matrix $A^{C}$ is considered in another way. Our definition of a triangular hesitant fuzzy matrix expresses the matrix $A^{C}$ in the subsequent steps:

- Step 1. Suppose $A^{L}=\left[a_{i j}^{L}\right]_{m \times n}$ and $A^{U}=\left[a_{i j}^{U}\right]_{m \times n}$ be two matrices such that $a_{i j}^{L}<a_{i j}^{U}$ for all $i$ and $j$.

- Step 2. Discuss the universe of the triangular hesitant fuzzy matrix as the set $L_{1}=\{A: \exists \lambda \in \mathbb{R}: A=$ $\left.\lambda A^{L}+w(1-\lambda) A^{U}\right\}$. It can be easily seen that the line $L_{1}$ is produced by the two matrices $A^{L}=\left[a_{i j}^{L}\right]_{m \times n}$ and $A^{U}=\left[a_{i j}^{U}\right]_{m \times n}$ in the space of $m \times n$ matrices.

- Step 3. State the support of the triangular hesitant fuzzy matrix to form the interval of the matrix: $\left[A^{L}, A^{U}\right]=$ $\left\{A: A=\lambda A^{L}+w(1-\lambda) A^{U}\right\}$.

- Step 4. Compute a matrix $A^{C}$ such that $A^{C} \in\left[A^{L}, A^{U}\right]$. In Step 3, by definition of interval $\left[A^{L}, A^{U}\right]$ it easily confirms that there exists a number $\bar{\lambda}$ such that $\left.A^{C}=\bar{\lambda} A^{L}+w(1-\bar{\lambda}) A^{U}\right\}$. Here, $\bar{\lambda}$ is independent on $i$ and $j$ such that the matrix $A^{C}$ lies on the interval of matrices $\left[A^{L}, A^{U}\right]$.

- Step 5. From Step 1 to Step 4, we consider the triangular hesitant fuzzy matrix as follows: $\tilde{\tilde{A}}=\left[\tilde{\tilde{a}}_{i j}\right]_{m \times n}=$ $\left(A^{L}, A^{C}, A^{U}\right)$ with the membership function is defined in (2.5).

\section{Problem environment}

First, we reduce the initial hesitant fuzzy MADM problem into a two-person game with hesitant fuzzy payoffs and thereafter we solve the formulated MADM game by LPP and HHFOWA-TOPSIS approach. This MADM 
TABLE 3. Hesitant fuzzy MADM problem.

\begin{tabular}{cccccc}
\hline \hline & & $C_{1}$ & $C_{2}$ & $\ldots$ & $C_{n}$ \\
\hline & $A_{1}$ & $\tilde{\tilde{a}}_{11}$ & $\tilde{\tilde{a}}_{12}$ & $\ldots$ & $\tilde{\tilde{a}}_{1 n}$ \\
$\tilde{\tilde{A}}=$ & $A_{2}$ & $\tilde{\tilde{a}}_{21}$ & $\tilde{\tilde{a}}_{22}$ & $\ldots$ & $\tilde{\tilde{a}}_{2 n}$ \\
$\vdots$ & $\vdots$ & $\vdots$ & $\ldots$ & $\vdots$ \\
& $A_{m}$ & $\tilde{\tilde{a}}_{m 1}$ & $\tilde{\tilde{a}}_{m 2}$ & $\ldots$ & $\tilde{\tilde{a}}_{m n}$ \\
\hline
\end{tabular}

game is converted into two-person crisp MADM game by using $\alpha$-cut and maximin principle, and then it is solved by LPP. Also the initial hesitant fuzzy MADM game is solved by HHFOWA approach for comparison purpose. The $\alpha$-cut is emerged in the procedure of Sakawa and Yano [45] for analyzing multi-objective non-linear problem with fuzzy information.

\subsection{Statement of the problem}

Now we present a hesitant fuzzy MADM problem which is described in Table 3 as:

Where the player I has $m$ alternatives $\left(A_{i}, i=1,2, \ldots, m\right)$ corresponding to $n$ attributes $\left(C_{j}, j=1,2, \ldots, n\right)$; $\tilde{\tilde{a}}_{i j}$ stands for ranking of $i$-th alternative corresponding to $j$-th attribute. The main intention of player I is to choose the best alternative in accordance with the obtainable information in the hesitant fuzzy MADM problem. Here, player I assumes that $\tilde{\tilde{A}}=\left[\tilde{\tilde{a}}_{i j}\right]_{m \times n}=\left(A^{L}, A^{C}, A^{U}\right)$ is a hesitant fuzzy MADM problem.

\subsection{Model formulation}

Here, we convert the basic hesitant fuzzy MADM problem into two-person zero-sum game by using $\alpha$-cut and maximin principle. Now we consider the following subsection for describing our approach.

\subsubsection{The $\alpha$-cut}

From the approach of Sakawa and Yano [45], player I applies the concept of $\alpha$-cut into the matrix $\tilde{\tilde{A}}=$ $\left\{A: \mu_{\tilde{\tilde{A}}}(A) \geq \alpha\right\}$. Then $\mu(\lambda)$ in equation (2.5) is just the membership function of the triangular hesitant fuzzy number $\tilde{\tilde{a}}=(0, \bar{\lambda}, 1), \bar{\lambda} \in[0,1]$. From the characteristics of triangular hesitant fuzzy number, $\alpha$-cut is connected with this hesitant fuzzy number, which will be an interval type $\{\lambda: \lambda \in \mathbb{R}, \mu(\lambda) \geq \alpha\}=\left[\lambda_{\alpha}^{L}, \lambda_{\alpha}^{U}\right]$, where $\lambda_{\alpha}^{L}$ and $\lambda_{\alpha}^{U}$ are lower and upper bounds of the $\alpha$-cut respectively. Here, we assume that $A_{\alpha}^{L}=\lambda_{\alpha}^{L} A^{L}+w\left(1-\lambda_{\alpha}^{L}\right) A^{U}$ and $A_{\alpha}^{U}=\lambda_{\alpha}^{U} A^{L}+w\left(1-\lambda_{\alpha}^{U}\right) A^{U} . A_{\alpha}^{L}=\left[a_{\alpha i j}^{L}\right]_{m \times n}$ and $A_{\alpha}^{U}=\left[a_{\alpha i j}^{U}\right]_{m \times n}$ are the $\alpha$-cut lower and upper bound matrices respectively, connected with the hesitant fuzzy matrix $\tilde{\tilde{A}}$. From the $\alpha$-cut, connected with the triangular hesitant fuzzy number $\tilde{\tilde{a}}=(0, \bar{\lambda}, 1)$, it can be expressed as $\left[\lambda_{\alpha}^{L}, \lambda_{\alpha}^{U}\right]=\{\lambda: \exists \delta \in[0,1]$ : $\left.\lambda=\delta \lambda_{\alpha}^{L}+w(1-\lambda) \lambda_{\alpha}^{U}\right\}$. Then we choose the relation between the membership functions $\mu(\lambda)$ and $\mu_{\tilde{\tilde{A}}}(A)$ is $\mu(\lambda)=\mu_{\tilde{\tilde{A}}}(A)=\mu_{\tilde{\tilde{A}}}\left(\lambda A^{U}+w(1-\lambda) A^{L}\right)$. Now the $\alpha$-cut set $\tilde{\tilde{A}}_{\alpha}=\left\{A: \mu_{\tilde{\tilde{A}}}(A) \geq \alpha\right\}$ connected with the triangular hesitant fuzzy matrix $\tilde{\tilde{A}}=\left(A^{L}, A^{C}, A^{U}\right)$ can be stated as:

$$
\tilde{\tilde{A}}_{\alpha}=\left\{\bar{A}: \bar{A} \in L_{1}, \mu_{\tilde{\tilde{A}}}(\bar{A}) \geq \alpha\right\}=\left[A_{\alpha}^{L}, A_{\alpha}^{U}\right]=\left\{\lambda A_{\alpha}^{U}+w(1-\lambda) A_{\alpha}^{L}\right\} .
$$

In this way we achieve an interval MADM decision matrix with the help of equation (3.1), which is shown in Table 4.

\subsubsection{Two-person zero-sum game}

Now, we have an interval MADM decision matrix (i.e., Table 4). Player I does not have any message over the feature of this decision matrix due to uncertainty. Here we reduce the interval MADM decision matrix to a twoperson zero-sum game. So player I has to accept one of the criteria i.e., the maximin principle of decision making 
TABLE 4. Interval MADM decision matrix.

\begin{tabular}{|c|c|c|c|c|c|}
\hline & & $C_{1}$ & $C_{2}$ & & $C_{n}$ \\
\hline \multirow[t]{2}{*}{$\bar{A}=$} & $\begin{array}{l}A_{1} \\
A_{2}\end{array}$ & $\begin{array}{l}{\left[a_{11}^{L}, a_{11}^{U}\right]} \\
{\left[a_{21}^{L}, a_{21}^{U}\right]}\end{array}$ & $\begin{array}{l}{\left[a_{12}^{L}, a_{12}^{U}\right]} \\
{\left[a_{22}^{L}, a_{22}^{U}\right]}\end{array}$ & $\begin{array}{l}\cdots \\
\cdots\end{array}$ & $\begin{array}{l}{\left[a_{1 n}^{L}, a_{1 n}^{U}\right]} \\
{\left[a_{2 n}^{L}, a_{2 n}^{U}\right]}\end{array}$ \\
\hline & $\dot{A_{m}}$ & {$\left[a_{m 1}^{L}, a_{m 1}^{U}\right]$} & {$\left[a_{m 2}^{L}, a_{m 2}^{U}\right]$} & $\begin{array}{l}\cdots \\
\cdots \\
\end{array}$ & {$\left[a_{m n}^{L}, a_{m n}^{U}\right]$} \\
\hline
\end{tabular}

TABLE 5. Two-person zero-sum crisp matrix game.

\begin{tabular}{ccccc}
\hline \hline & $C_{1}$ & $C_{2}$ & $\ldots$ & $C_{n}$ \\
\hline \multirow{4}{*}{$A=$} & $a_{11}$ & $a_{12}$ & $\ldots$ & $a_{1 n}$ \\
$A_{2}$ & $a_{21}$ & $a_{22}$ & $\ldots$ & $a_{2 n}$ \\
$\vdots$ & $\ldots$ & $\ldots$ & $\ldots$ & $\ldots$ \\
$A_{m}$ & $a_{m 1}$ & $a_{m 2}$ & $\ldots$ & $a_{m n}$ \\
\hline
\end{tabular}

problem in fuzziness. According to the fact, player I is protective and conclude that player II is against him. So from equation (3.1), we see that interval MADM decision matrix form in a two-person zero-sum game versus player II. Now we try to find the expected payoffs and optimal solutions of player I and player II. Player I views player II as non-cooperative player. Then we consider $X$ i.e., $X=\left\{x: \sum_{i=1}^{m} x_{i}=1, x_{i} \geq 0, i=1,2, \ldots, m\right\}$ and $Y=\left\{y: \sum_{j=1}^{n} y_{j}=1, y_{j} \geq 0, j=1,2, \ldots, n\right\}$ are the pair of mixed strategies of player I and player II over the collection of alternatives $\left\{A_{i}, i=1.2, \ldots, m\right\}$ and the collection of attributes $\left\{C_{j}, j=1,2, \ldots, n\right\}$ respectively. Considering the various values of $\alpha$ in the $\alpha$-cut, $\tilde{\tilde{A}}_{\alpha}=\left\{\lambda A_{\alpha}^{U}+w(1-\lambda) A_{\alpha}^{L}, \lambda \in[0,1]\right\}$ given in equation (3.1). Then we make two-person zero-sum crisp matrix game which is shown in Table 5 .

Player I is the maximizing player and player II is the minimizing player. From definition, the expected payoff of the player I is $x^{t} A y=\sum_{j=1}^{n} \sum_{i=1}^{m} a_{i j} x_{i} y_{j}$ and for player II is $-x^{t} A y$. So the basic hesitant fuzzy MADM problem is reduced into matrix game with crisp payoffs. Now we derive the optimal strategies of player I and player II and the value of the game.

\section{SOLUTION PROCEDURE}

In this section, we give a detailed discussion of LPP and HHFOWA-TOPSIS approach. The procedure for solving the hesitant fuzzy MADM game consists of two algorithms. Algorithm 4.6 is for obtaining the optimal strategy of crisp MADM game and ranking order of each alternative. Then Algorithm 4.7 is based on HHFOWATOPSIS approach to get the optimal ranking order of alternatives.

\subsection{LPP}

At first we convert a two-person crisp matrix game (i.e., Table 5) into a problem for solving two free optimization problems. Then by Theorem 1, to find a solution for crisp game, then we derive the optimal solution of the consequent pair of optimization models and finally we obtain the mixed strategies. The consequent pair of optimization models is as follows:

$$
\begin{array}{cc}
(\mathbf{M O D}-\mathbf{5}) \min _{x^{\prime}} x^{\prime t} e_{m} & (\mathbf{M O D}-\mathbf{6}) \max _{y^{\prime}} y^{\prime t} e_{n} \\
\text { subject to }\left\{\begin{array}{l}
x^{\prime} t A_{\alpha} \geq e_{n}^{t} \\
x_{i}^{\prime} \geq 0, \forall i
\end{array}\right. & \text { subject to }\left\{\begin{array}{l}
A_{\alpha} y^{\prime} \leq e_{m} \\
y_{j}^{\prime} \geq 0, \forall j
\end{array}\right.
\end{array}
$$


where $A_{\alpha}=\left[a_{\alpha i j}\right] \in \tilde{\tilde{A_{\alpha}}}$. So we have the following definition:

Definition 4.1. Let $x_{\alpha}^{\prime *}$ and $y_{\alpha}^{\prime *}$ are the optimal solutions of (MOD-5) and (MOD-6). Then the mixed strategies $x_{\alpha}^{*}=v_{1} x_{\alpha}^{\prime *}$ and $y_{\alpha}^{*}=v_{2} y_{\alpha}^{*}$, where $\sum_{i=1}^{m} x_{i}^{\prime *}=\frac{1}{v_{1}}$ and $\sum_{j=1}^{n} y_{j}^{\prime *}=\frac{1}{v_{2}}$ are said to be optimal strategies of player I and player II respectively.

Corollary 4.2. (MOD-5) and (MOD-6) are equivalent to the following models respectively,

$$
\begin{array}{rr}
\left(\text { MOD - 7) } \min _{x^{\prime}} x^{\prime} e_{m}\right. & (\text { MOD }-8) \max _{y^{\prime}} y^{\prime t} e_{n} \\
\text { subject to }\left\{\begin{array}{l}
x^{\prime} A_{\alpha}^{U} \geq e_{n} \\
x^{\prime} t A_{\alpha}^{L} \geq e_{n} \\
x_{i}^{\prime} \geq 0, \forall i .
\end{array}\right. & \text { subject to }\left\{\begin{array}{l}
A_{\alpha}^{U} y^{\prime} \leq e_{m} \\
A_{\alpha}^{L} y^{\prime} \leq e_{m} \\
y_{j}^{\prime} \geq 0, \forall j .
\end{array}\right.
\end{array}
$$

Proof. We want to prove that (MOD-5) and (MOD-7) are same. So our problem is

$($ MOD - 9)

$$
\begin{gathered}
\min _{x^{\prime}} x^{\prime}{ }^{t} e_{m} \\
\text { subject to } x^{\prime t} A_{\alpha} \geq e_{n} \\
x_{i}^{\prime} \geq 0, \forall i, A_{\alpha}=\left[a_{\alpha i j}\right] \in \tilde{\tilde{A}}_{\alpha}
\end{gathered}
$$

since $A_{\alpha} \in \tilde{\tilde{A_{\alpha}}}$, it can be expressed as $\left\{\eta A_{\alpha}^{L}+w(1-\eta) A_{\alpha}^{U}\right\}, 0 \leq \eta \leq 1$, then (MOD-9) can be stated as (Model-10).

$$
\begin{array}{r}
\left(\text { MOD - 10) } \min _{x^{\prime}} x^{\prime t} e_{m}\right. \\
\text { subject to }\left\{\begin{array} { l } 
{ x ^ { \prime } { } ^ { \prime } P + \eta x ^ { \prime } t Q \geq e _ { n } } \\
{ x _ { i } ^ { \prime } \geq 0 , \forall i } \\
{ 0 \leq \eta \leq 1 . }
\end{array} \text { subject to } \left\{\begin{array}{l}
x^{\prime}{ }^{\prime} P \geq e_{n} \\
x^{\prime}{ }^{\prime} P+x^{\prime} Q \geq e_{n} \\
x_{i}^{\prime} \geq 0, \forall i .
\end{array}\right.\right.
\end{array}
$$

where $P=A_{\alpha}^{U}$, and $Q=w\left(A_{\alpha}^{L}-A_{\alpha}^{U}\right)$. We want to show that set of constraints of (MOD-10) and (MOD-11) are same. Let us consider that $x^{\prime}$ justifies the constraints of (MOD-11), then we get

$$
x^{\prime t} P \geq e_{n} \text { and } x^{\prime t} P+x^{\prime t} Q \geq e_{n}, \quad x_{i}^{\prime} \geq 0 .
$$

So we multiply the first inequality by $w(1-\eta)$ and second by $w \eta$, with $\eta \in[0,1]$, we have $w(1-\eta) x^{\prime t} P \geq$ $w(1-\eta) e_{n}$ and $w \eta x^{\prime t} P+w \eta x^{\prime t} Q \geq w \eta e_{n}, x_{i}^{\prime} \geq 0$. Adding these two inequalities we get $x^{\prime}{ }^{\prime} P+\eta x^{\prime}{ }^{t} Q \geq e_{n}$ with $x_{i}^{\prime} \geq 0$ and $0 \leq \eta \leq 1$. So, $x^{\prime}$ justifies the constraints of (MOD-10). Now we observe that $x^{\prime}$ satisfies the constraints of (MOD-11), if we take $\eta=0$ and $\eta=1$ in the constraints of (MOD-10). Thus the objective functions are identical in (MOD-10) and (MOD-11). Hence we can state that the two problems are same. Also, if we replace $P$ and $Q$ with $w\left(A_{\alpha}^{U}\right)$ and $w\left(A_{\alpha}^{L}-A_{\alpha}^{U}\right)$ respectively, the expected similarity will be checked. Similarly the equality of (MOD-6) and (MOD-8) can be verified. Then by Corollary 4.2 we convert the (MOD-5) and (MOD-6) to the simple LPPs (MOD-7) and (MOD-8) and then solve easily using the simplex method.

\subsubsection{Score function}

Now we propose a new score function to determine the expected score of alternatives $A_{i}(i=1,2, \ldots, m)$ corresponding to the attributes $C_{j}(j=1,2, \ldots, n)$, associated with the decision matrix. Score function plays 
a significant part in real-life decision making problem concerning imprecision or uncertain information and specially in decision making problem. Many types of score functions are chosen to solve such problems. Here we design a new score function for the initial hesitant fuzzy MADM problem. Now we define the expected score for an alternative in MADM game.

Definition 4.3. Let $x_{\alpha}^{\prime *}$ and $y_{\alpha}^{\prime *}$ are the optimal solutions of (MOD-5) and (MOD-6), respectively, so the desired score of every alternative $A_{i}$ corresponding to the decision matrix $\tilde{\tilde{A}}=\left[\tilde{\tilde{a}}_{i j}\right] \in \tilde{\tilde{A}}_{\alpha}$, and $\alpha$-cut is denoted by $E S\left(A_{i}, \tilde{\tilde{A}}\right)$ and defined as $E S\left(A_{i}, \tilde{\tilde{A}}\right)=x_{i, \alpha}^{*} \sum_{j=1}^{n} a_{i j} y_{j, \alpha}^{*}$, where $x_{i, \alpha}^{*}$ and $y_{j, \alpha}^{*}$ are the parts of the mixed strategies $x_{\alpha}^{*}$ and $y_{\alpha}^{*}$, respectively, those are determined by the following relation $x_{\alpha}^{*}=x_{\alpha}^{*} / v_{1}$ and $y_{\alpha}^{*}=y_{\alpha}^{*} / v_{2}$, and $\sum_{i=1}^{m} x_{i, \alpha}^{* *}=\frac{1}{v_{1}}$ and $\sum_{j=1}^{n} y_{j, \alpha}^{* *}=\frac{1}{v_{2}}$.

Corollary 4.4. Let $A_{\alpha}=\left[a_{\alpha i j}\right]$ be a matrix in $\tilde{\tilde{A}}_{\alpha}$, the expected score of each alternative $A_{i}, \operatorname{ES}\left(A_{i}, A_{\alpha}\right)=$ $x_{i, \alpha}^{*} \sum_{j=1}^{n} a_{\alpha i j} y_{j, \alpha}^{*}$ in $A_{\alpha}$ satisfies the following relation:

$$
x_{i, \alpha}^{*} \sum_{j=1}^{n} a_{\alpha i j}^{L} y_{j, \alpha}^{*} \leq E S\left(A_{i}, A_{\alpha}\right) \leq x_{i, \alpha}^{*} \sum_{j=1}^{n} a_{\alpha i j}^{U} y_{j, \alpha}^{*} .
$$

Proof. We have $a_{i j}^{L} \leq a_{\alpha i j} \leq a_{i j}^{U}$ for all $i$ and $j$. Therefore, the proof is straightforward.

Corollary 4.5. Using Corollary 4.4, we calculate the range $\left[x_{i, \alpha}^{*} \sum_{j=1}^{n} a_{\alpha i j}^{L} y_{j, \alpha}^{*}, x_{i, \alpha}^{*} \sum_{j=1}^{n}\right.$

$\left.a_{\alpha i j}^{U} y_{j, \alpha}^{*}\right]$ of the desired score of every alternative $A_{i}$ in MADM problem with decision matrix $A_{\alpha} \in \tilde{\tilde{A}}_{\alpha}$, then we derive only the optimal solutions of (MOD-7) and (MOD-8).

New score function based on hesitant fuzzy number:

Applying interval ordering, the ideal way to rank the alternatives is to rank the interval $\left[x_{i, \alpha}^{*} \sum_{j=1}^{n} a_{\alpha i j}^{L} y_{j, \alpha}^{*}, x_{i, \alpha}^{*} \sum_{j=1}^{n} a_{\alpha i j}^{U} y_{j, \alpha}^{*}\right], \quad i=1,2, \ldots, m$. But we have no surety about the overlapping of these intervals. To cover this situation, we consider a pointwise comparison of every interval. We put weights $\psi$ and $1-\psi$ with $\psi \in[0,1]$, then determine the score of alternatives $A_{i}, i=1,2, \ldots, m$ as:

$$
A_{i}=\psi\left(x_{i, \alpha}^{*} \sum_{j=1}^{n} a_{\alpha i j}^{U} y_{j, \alpha}^{*}\right)+w(1-\psi)\left(x_{i, \alpha}^{*} \sum_{j=1}^{n} a_{\alpha i j}^{L} y_{j, \alpha}^{*}\right) .
$$

As for example, player I chooses $\psi=0$ if he tries to compare the lower value of intervals and if player I tries to compare the intervals by the middle value then he selects $\psi=0.5$. Then the weight $\psi \in[0,1]$ stands for the significance if player I gives on upper expected value. So the weight $(1-\psi)$ describes the significance pointed to the lower expected value. Then player I chooses the weight $\psi \in[0,1]$ to calculate the expected score of each alternative $A_{i}$ associated with the $\alpha$-cut by equation (4.1), where $\alpha$ is chosen by player I. Using the new score function, player I can rank the alternatives from the highest to the lowest based on the varying values of $\alpha$ and $\psi$.

Algorithm 4.6. Now we develop an algorithm to find the optimal strategies of player I and player II. Also we find the expected score of each alternative corresponding to the attributes. In this procedure, player I decides the $\alpha$-cut based on the chosen value $\alpha$.

- Step 1. Player I selects the $\alpha$-cut to obtain the optimal solutions $x_{\alpha}^{\prime *}$ and $y_{\alpha}^{\prime *}$ of (MOD-5) and (MOD-6), respectively consequently determines the mixed strategies $x_{\alpha}^{*}=v_{1} x_{\alpha}^{*}$ and $y_{\alpha}^{*}=v_{2} y_{\alpha}^{*}$. Definition 4.1 and Corollary 4.2 show that these strategies are the optimal solutions of the two-person zero-sum game. 
- Step 2. Player I considers the weight $\psi \in[0,1]$, and then determines the desired score of every alternative according to equation (4.1).

- Step 3. At last, we present the preference order of each alternative according as first (1), second (2), third (3) and fourth (4).

\subsection{The proposed HHFOWA-TOPSIS approach}

In this subsection, we propose the TOPSIS approach based on HHFOWA operator. The basic principle of the proposed TOPSIS approach is the chosen alternative should have the maximal and minimal hybrid hesitant fuzzy normalized Euclidean distance from PIS and NIS respectively.

Suppose that an MADM problem has $m$ alternatives $\left(A_{i}, i=1,2, \ldots, m\right)$ corresponding to $n$ attributes $\left(C_{j}, j=\right.$ $1,2, \ldots, n)$. All the values are assigned to the alternatives with hesitant fuzzy attribute can be expressed in a decision matrix. So the procedure of HHFOWA-TOPSIS approach is discussed in Algorithm 4.7.

Algorithm 4.7. - Step 1. Calculate the PIS $\tilde{\tilde{\tilde{h}}}_{\tilde{\tilde{h}}}\left(A^{+}\right)$and NIS $\left(A^{-}\right)$respectively as $A^{+}=\left\{\tilde{\tilde{h}}_{1}^{+}, \tilde{\tilde{h}}_{2}^{+}, \ldots, \tilde{\tilde{h}}_{n}^{+}\right\}$and $A^{-}=\left\{\tilde{\tilde{h}}_{1}^{-}, \tilde{\tilde{h}}_{2}^{-}, \ldots, \tilde{\tilde{h}}_{n}^{-}\right\}$, where $\tilde{\tilde{h}}_{j}^{+}=\cup_{i=1}^{m} \tilde{\tilde{h}}_{i j}=\cup_{\gamma_{1 j} \in \tilde{\tilde{h}}_{i j}, \ldots, \gamma_{m j} \in \tilde{\tilde{h}}_{m j}} \max \left(\gamma_{i j}, \ldots, \gamma_{m j}\right)$ and $\tilde{\tilde{h}}_{j}^{-}=\cap_{i=1}^{m} \tilde{\tilde{h}}_{i j}=$ $\cap_{\gamma_{1 j} \in \tilde{h}_{i j}, \ldots, \gamma_{m j} \in \tilde{\tilde{h}}_{m j}} \min \left(\gamma_{i j}, \ldots, \gamma_{m j}\right), j=1,2, \ldots, n$.

- Step 2. Determine the HHFOWA between each alternative with the hesitant fuzzy PIS and the hesitant fuzzy NIS by using Definition 2.11 as follows:

$$
\begin{gathered}
D_{i}^{+}=\operatorname{HhFOwA}\left(A_{i}, A^{+}\right)=\sum_{j=1}^{n} w_{j} d\left(h_{i j}, h_{j}{ }^{+}\right) \\
D_{i}^{-}=\operatorname{HhfOWA}\left(A_{i}, A^{-}\right)=\sum_{j=1}^{n} w_{j} d\left(h_{i j}, h_{j}{ }^{-}\right) .
\end{gathered}
$$

- Step 3. The relative closeness of the alternatives $A_{i}$ can be defined as:

$$
D_{i}=\frac{\operatorname{HHFOWA}\left(A_{i}, A^{-}\right)}{\operatorname{HHFOWA}\left(A_{i}, A^{-}\right)+\operatorname{HHFOWA}\left(A_{i}, A^{+}\right)}, i=1,2, \ldots, m .
$$

- Step 4. Then rank the alternatives according to their scores.

\section{Application example}

An investment company is wishing to invest a lump-sum amount of money in one of possible four alternatives which are as: the first alternative $\left(A_{1}\right)$ is a TV company, the second alternative $\left(A_{2}\right)$ is a computer company, the third alternative $\left(A_{3}\right)$ is a food company and the fourth alternative $\left(A_{4}\right)$ is a car company. The investment company also suggests five attributes based on the alternatives and they are: production risk $\left(C_{1}\right)$, financial risk $\left(C_{2}\right)$, management risk $\left(C_{3}\right)$, environment risk $\left(C_{4}\right)$ and market risk $\left(C_{5}\right)$. The triangular hesitant fuzzy decision matrix $\tilde{\tilde{A}}=\left[\tilde{\tilde{a}}_{i j}\right]_{m \times n}=\left(A^{L}, A^{C}, A^{U}\right)($ set $\bar{\lambda}=0.5)$ is obtained from the investment selection problem which is as follows:

$$
\begin{aligned}
& A^{L}=\left[\begin{array}{lllll}
0.25 & 0.15 & 0.85 & 0.65 & 0.45 \\
0.65 & 0.35 & 0.15 & 0.45 & 0.25 \\
0.15 & 0.75 & 0.15 & 0.45 & 0.15 \\
0.35 & 0.45 & 0.55 & 0.35 & 0.55
\end{array}\right], A^{C}=\left[\begin{array}{lllll}
0.373 & 0.278 & 0.875 & 0.753 & 0.495 \\
0.708 & 0.423 & 0.278 & 0.563 & 0.328 \\
0.233 & 0.801 & 0.323 & 0.518 & 0.278 \\
0.468 & 0.518 & 0.613 & 0.423 & 0.658
\end{array}\right] \\
& A^{U}=\left[\begin{array}{lllll}
0.55 & 0.45 & 1.00 & 0.95 & 0.60 \\
0.85 & 0.55 & 0.45 & 0.75 & 0.45 \\
0.35 & 0.95 & 0.55 & 0.65 & 0.45 \\
0.65 & 0.65 & 0.75 & 0.55 & 0.85
\end{array}\right]
\end{aligned}
$$

Here we consider the weight $w=0.9$ so that the center value $\left(A^{C}\right)$ lies between $A^{L}$ and $A^{U}$. 
TABLE 6. Computational results of $x_{\alpha}^{*}$ and $y_{\alpha}^{*}$.

\begin{tabular}{ccccc}
\hline \hline$x_{\alpha}^{*}$ and $y_{\alpha}^{*}$ & $\alpha=0.3$ & $\alpha=0.5$ & $\alpha=0.7$ & $\alpha=0.9$ \\
\hline$x_{1, \alpha}^{*}$ & 0.09 & 0.08 & 0.08 & 0.08 \\
$x_{2, \alpha}^{*}$ & 0.30 & 0.26 & 0.30 & 0.29 \\
$x_{3, \alpha}^{*}$ & 0.11 & 0.11 & 0.10 & 0.12 \\
$x_{4, \alpha}^{*}$ & 0.50 & 0.55 & 0.52 & 0.51 \\
$y_{1, \alpha}^{*}$ & 0.17 & 0.24 & 0.28 & 0.18 \\
$y_{2, \alpha}^{*}$ & 0.28 & 0.33 & 0.34 & 0.28 \\
$y_{3, \alpha}^{*}$ & 0.00 & 0.13 & 0.18 & 0.00 \\
$y_{4, \alpha}^{*}$ & 0.35 & 0.00 & 0.20 & 0.35 \\
$y_{5, \alpha}^{*}$ & 0.19 & 0.30 & 0.00 & 0.19 \\
\hline
\end{tabular}

TABLE 7. Score of each hesitant fuzzy alternative with its priority.

\begin{tabular}{ccccc}
\hline \hline & $A_{1}$ & $A_{2}$ & $A_{3}$ & $A_{4}$ \\
\hline$\alpha=\psi=0.3$ & $0.043(4)$ & $0.144(2)$ & $0.053(3)$ & $0.241(1)$ \\
$\alpha=\psi=0.5$ & $0.034(4)$ & $0.109(2)$ & $0.046(3)$ & $0.227(1)$ \\
$\alpha=\psi=0.7$ & $0.038(4)$ & $0.143(2)$ & $0.048(3)$ & $0.247(1)$ \\
$\alpha=\psi=0.9$ & $0.040(4)$ & $0.144(2)$ & $0.532(3)$ & $0.249(1)$ \\
\hline
\end{tabular}

\subsection{Results and discussion}

To solve this problem, we apply the LPP and HHFOWA-TOPSIS approach to obtain the optimal ranking order.

\subsubsection{Applying the LPP}

We impose the proposed Algorithm 4.6 to validate the MADM game. First, the $\alpha$-cut is fixed for different values within the interval $[0,1]$, and then calculate the optimal strategies $x_{\alpha}^{*}$ and $y_{\alpha}^{*}$ which are shown in Table 6 (by Step 1 of Algorithm 4.6). Secondly, player I sets the value of $\psi$. Then determine the expected score of every alternative (by Step 2 of Algorithm 4.6) corresponding to various $\alpha$-cuts and different $\psi$. Thereafter, the accepted outcomes are displayed in Table 7 . Now for this example, we say that the ranking of each alternative remains adjustable for various values of $\alpha$. Here, we choose: poor (0.3), medium (0.5), good (0.7) and excellent (0.9). At last, we give the preference order of each alternative by Step 3: $A_{4}$ (Car company) is considered as the best alternative with largest score and $A_{1}$ is a computer company, likes to be the worst alternative with the smallest score in this MADM game. So, the ranking order of this MADM game will be $A_{4}>A_{2}>A_{3}>A_{1}$ (> means "is preferred to"). So this example displays that the MADM game process can be applied for the investment selection problem.

\subsubsection{Applying HHFOWA-TOPSIS aproach}

The example concerning investment company is employed to compare the effectiveness of proposed HHFWOATOPSIS approach with the LPP. Suppose that the weight vector of the criteria is $W=(0.1,0.2,0.2,0,2,0.3)$. Now we employ Algorithm 4.7 to obtain the optimal ranking order. 
- Step 1. Using the score function, calculate the PIS $\left(A^{+}\right)$and NIS $\left(A^{-}\right)$respectively as:

$$
\begin{aligned}
A^{+} & =\left\{\begin{array}{c}
\{0.650,0.708,0.850\},\{0.750,0.801,0.950\},\{0.850,0.875,1.000\}, \\
\{0.650,0.753,0.950\},\{0.550,0.658,0.850\}
\end{array}\right\} \\
\text { and } A^{-} & =\left\{\begin{array}{c}
\{0.150,0.323,0.350\},\{0.150,0.2781,0.450\},\{0.150,0.278,0.450\}, \\
\{0.350,0.423,0.550\},\{0.150,0.278,0.450\}
\end{array}\right\}
\end{aligned}
$$

- Step 2. Using equations (4.2) and (4.3), to obtain the HHFOWA between each alternative with the hesitant fuzzy PIS and the hesitant fuzzy NIS respectively as:

$$
\begin{aligned}
D_{1}^{+}=\operatorname{HhFOWA}\left(A_{1}, A^{+}\right)=0.20915, D_{2}^{+}=\operatorname{HHFOWA}\left(A_{2}, A^{+}\right)=0.33436, \\
D_{3}^{+}=\operatorname{HhFOWA}\left(A_{3}, A^{+}\right)=0.35185, D_{4}^{+}=\operatorname{HHFOWA}\left(A_{4}, A^{+}\right)=0.20195, \\
D_{1}^{-}=\operatorname{HhFOWA}\left(A_{1}, A^{-}\right)=0.28774, D_{2}^{-}=\operatorname{HHFOWA}\left(A_{2}, A^{-}\right)=0.14475, \\
D_{3}^{-}=\operatorname{HhFOWA}\left(A_{3}, A^{-}\right)=0.15099, D_{4}^{-}=\operatorname{HHFOWA}\left(A_{4}, A^{-}\right)=0.28875 .
\end{aligned}
$$

- Step 3. The relative closeness of the alternatives $A_{i}$ is determined by the equation of Step 3 (Algorithm 4.6) as:

$$
\begin{gathered}
D_{i}=\frac{\operatorname{HHFOWA}\left(A_{i}, A^{-}\right)}{\operatorname{HHFOWA}\left(A_{i}, A^{-}\right)+\operatorname{HHFOWA}\left(A_{i}, A^{+}\right)}, i=1,2, \ldots, m . \\
\text { So } D_{1}=0.5790, D_{2}=0.3021, D_{3}=0.3002 \text { and } D_{4}=0.5885 .
\end{gathered}
$$

- Step 4. Then the ranking order of the alternatives is $A_{4}>A_{1}>A_{2}>A_{3}$. So the alternative $A_{4}$ is the best according to their scores.

\section{Comparisons}

In comparison with the related papers, the proposed study has the following advantages:

- It ought to be noted that our primary problem is a hesitant fuzzy MADM problem. In our content, $\alpha$-cuts are taken to defuzzify the hesitant fuzzy MADM problem for obtaining the crisp two-person zero-sum MADM game. Thereafter, LPP and HHFOWA-TOPSIS approach are applied to solve the hesitant fuzzy MADM game for deriving the optimal strategy and the expected score of each alternative corresponding to the attributes are different than Chen and Larbani [11].

- Sakawa and Yano [45] discussed the $\alpha$-cuts for solving multi-objective non-linear programming problems with fuzzy information. Again Sakawa and Yano [45] considered the minimax principle i.e., they chosen the decision variable that accepted values in the $\alpha$-cut but we consider maximin principle. Here we formulate the problem as a game versus Nature, and another procedure is introduced for solving hesitant fuzzy MADM game.

- Nishizaki and Sakawa [36] and Sakawa and Nishizaki [46] proposed a two-person zero-sum fuzzy and multiobjective games for conflict solution. The elements of the payoff matrix are taken as L-R fuzzy numbers. A degree of acquirement of each fuzzy goal is defined due to the degree of satisfication of a decision. Therefore, a maximin solution with connection to a degree of acquirement of the aggregated fuzzy goal is discussed. In Nishizaki and Sakawa [36] and Sakawa and Nishizaki [46], the authors considered the maximin rule when conduct with the satisfaction degree of goals but in this paper, it is in the crisp game. Also, for obtaining maximin strategy of any player, the authors of Nishizaki and Sakawa [36] and Sakawa and Nishizaki [46] determined a fractional LPP, but here LPP and HHFOWA-TOPSIS approach are used to solve the hesitant fuzzy MADM game.

- Campos [9] converted a two-person zero-sum fuzzy matrix game into a crisp LPP by using different approaches for ranking fuzzy numbers. Here we consider the hesitant fuzzy MADM game, and the solution procedure is different. The procedure is applied by Bector and Chandra ([4]) is same with Campos [9]. 
TABLE 8. Comparison among our approach and existing approaches.

\begin{tabular}{ll}
\hline \hline Existing papers & Solution procedure \\
\hline Bector and Chandra ([4]) & $\begin{array}{l}\text { Using the concept of duality in LPP, fuzzy matrix } \\
\text { game } \\
\text { reduces to the pair of crisp primal and dual LPPs }\end{array}$ \\
\hline Campos [9] & $\begin{array}{l}\text { Using ranking function to convert a } \\
\text { zero-sum fuzzy matrix game into a crisp LPP }\end{array}$ \\
\hline Chen and Larbani [11] & $\begin{array}{l}\text { Using } \alpha \text {-cut and maximin principle to formulate } \\
\text { fuzzy MADM problem } \\
\text { into two-person zero-sum game with uncertain } \\
\text { payoff matrix }\end{array}$ \\
\hline Sakawa and Yano [45] & $\begin{array}{l}\text { Using } \alpha \text {-cut for solving multi-objective non-linear } \\
\text { programming problems with fuzzy information }\end{array}$ \\
\hline Nishizaki and Sakawa $[36]$ & $\begin{array}{l}\text { Using maximin approach for solving a fractional } \\
\text { and Sakawa and Nishizaki }\end{array}$ \\
LPP & $\begin{array}{l}\text { Using } \alpha \text {-cut and maximin principle to convert } \\
\text { hesitant fuzzy MADM problem into crisp matrix } \\
\text { game, which is solved by LPP and analyzed the } \\
\text { This paper } \\
\text { result with the solution of hesitant fuzzy MADM } \\
\text { game by HHFOWAapproach }\end{array}$ \\
\hline
\end{tabular}

Actually, Bector and Chandra ([4]) discussed an unfamiliar duality idea in LPPs with fuzzy payoffs and due to defuzzification they converted a fuzzy matrix game to the pair of crisp primal and dual LPPs. The comparison between our approach and existing approaches is shown in Table 8 .

Moreover, in all mentioned approaches, the elements of the payoff matrix are fuzzy number but in our work, elements are hesitant fuzzy numbers which depend on the value of $\lambda$. Thus our proposed approaches can be applied to analyze not only a hesitant fuzzy MADM problem but also a two-person zero-sum game with the hesitant fuzzy payoffs.

\subsection{Pros and Cons of the Proposed approaches}

- The LPP and HHFOWA-TOPSIS approach based on the hybrid hesitant fuzzy normalized Euclidean distance measure are incorporated in this paper. Based on the context of the problem, HHFOWA-TOPSIS is very easy than the LPP to apply in real-life problem.

- It is less computational effort for solving hesitant fuzzy MADM game.

- The LPP and HHFOWA-TOPSIS approach are not only solved the hesitant fuzzy MADM game problem, but also the other decision making problem under uncertain environment.

- In this paper, the weights of each attribute are given for HHFOWA-TOPSIS. But in some real-life problems, the weights are always not known. So it is a questionable to select the proper weight for the decision making problem, can be treated as one of the drawbacks of the approach.

\section{Conclusion}

In conventional MADM game problem, the weight of every attribute identifies the substances of such type of difficulty. Also numerous methods are available for obtaining the weights. Many researchers has investigated the chance of applicability of two-person zero-sum game in MADM process. In this content, we have considered a hesitant fuzzy MADM problem; and then we have formulated it as a crisp MADM game by considering the $\alpha$-cut and maximin principle. The proposed approaches has been conveyed us to initiate a new way to 
define the membership function for triangular hesitant fuzzy numbers. Here we have applied the LPP and HHFOWA-TOPSIS approach to solve the hesitant fuzzy MADM game problem. Two algorithms have been introduced to check the efficiency of application in this paper. An example of investment company has been shown the applicability and feasibility of using such MADM game. Based on the discussion, we have concluded that the HHFOWA-TOPSIS approach has been allowed more suitable to obtain the optimal ranking order of the alternatives. In practical situation, a large number of MADM games can be analyzed frequently and successfully by using the proposed approaches.

Future studies can be extended by considering the proposed approaches in the following directions:

- In this work, we have discussed the proposed approaches to the hesitant fuzzy MADM game. Further, the notion of proposed approaches can be utilized for analyzing the interval-valued hesitant, dual hesitant, Pythagorean hesitant fuzzy MADM problem, Lotfi et al. [31], Lotfi et al. [32], Lotfi et al. [29] and Lotfi et al. [30].

- Also aggregation operator is an effective tool to make the decision. Then the proposed TOPSIS can be extended for any other aggregation operator.

Acknowledgements. The research, Jishu Jana is partially supported by the Council of Scientific \& Industrial Research (CSIR) under JRF scheme with sanctioned no. 09/599(0067)/2016-EMR-I dated 20/10/2016. The authors are very much thankful to the respected Editor-in-Chief and anonymous reviewers for their valuable suggestions and comments, which helped us to improve the quality of the paper.

Compliance with ethical standards.

Conflict of interest. The authors do not have any possible conflicts of interest.

Ethical approval. This article does not contain any studies with human participants or animals performed by any of the authors.

\section{REFERENCES}

[1] F. Achemine, A. Merakeb, M. Larbani and P. Marthon, Z-equilibria in bi-matrix games with uncertain payoffs. RAIRO Oper. Res. 54 (2020) 393-412.

[2] M. Aggarwal, Hesitant information sets and application in group decision making. Appl. Soft Comput. 75 (2019) $120-129$.

[3] A. Aggarwal and I. Khan, Solving multi-objective fuzzy matrix games via multi-objective linear programming approach. Kybernetika 52 (2016) 153-168.

[4] C.R. Bector, S. Chandra and V. Vijay, Duality in linear programming with fuzzy parameters and matrix games with fuzzy payoffs. Fuzzy Sets Syst. 46 (2004) 253-269.

[5] A. Bhaumik and S.K. Roy, Intuitionistic interval-valued hesitant fuzzy matrix games with a new aggregation operator for solving management problem. Granul. Comput. 6 (2021) 359-375.

[6] A. Bhaumik, S.K. Roy and D.F. Li, Analysis of triangular intuitionistic fuzzy matrix games using robust ranking. J. Intell. Fuzzy Syst. 33 (2017) 327-336.

[7] A. Bhaumik, S.K. Roy and G.W. Weber, Hesitant interval-valued intuitionistic fuzzy-linguistic term set approach in Prisoners' dilemma game theory using TOPSIS: a case study on Human-trafficking. Cent. Eur. J. Oper. Res. 28 (2020) $797-816$.

[8] H. Bigdeli and H. Hassanpour, An approach to solve multi-objective linear production planning games with fuzzy parameters. Yugosl. J. Oper. Res. 28 (2018) 237-248.

[9] L. Campos, Fuzzy linear programming models to solve fuzzy matrix games. Fuzzy Sets Syst. 32 (1989) $275-289$.

[10] S.J. Chen and C.L. Hwang, Fuzzy Multiple Attribute Decision Making: Methods and Applications, Springer, New York (1992).

[11] Y.W. Chen and M. Larbani, Two-person zero-sum game approach for fuzzy multiple attribute decision making problems. Fuzzy Sets Syst. 157 (2006) 34-51.

[12] P.E. Ezimadu, Modelling cooperative advertising decisions in a manufacturer-distributor-retailer supply chain using game theory. Yugosl. J. Oper. Res. 30 (2020) 147-176.

[13] B. Farhadinia and Z.S Xu, A novel distance-based multiple attribute decision-making with hesitant fuzzy sets. Soft Comput. 24 (2020) 5005-5017.

[14] A. Hatami-Marbini and F. Kangi, An extension of fuzzy TOPSIS for a group decision making with an application to Tehran stock exchange. Appl. Soft Comput. 52 (2017) 1084-1097.

[15] C.L. Hwang and K. Yoon, Multiple attribute decision making methods and applications, Springer-Verlag, New York (1981).

[16] J. Jana and S.K. Roy, Solution of matrix games with generalized trapezoidal fuzzy payoffs. Fuzzy Inf. Eng. 10 (2018) $213-224$.

[17] J. Jana and S.K. Roy, Dual hesitant fuzzy matrix games: based on new similarity measure. Soft Comput. 23 (2019) 8873-8886.

[18] J. Jana and S.K. Roy, Soft matrix game: A hesitant fuzzy MCDM approach. Am. J. Math. Manag. Sci. 40 (2021) $107-119$. 
[19] L.S. Jin, R. Mesiar and R. Yager, Ordered weighted averaging aggregation on convex poset. IEEE Trans. Fuzzy Syst. 27 (2019) 612-617.

[20] S. Khalilpourazari and H.H. Doulabi, Designing a hybrid reinforcement learning based algorithm with application in prediction of the COVID-19 pandemic in Quebec. Ann. Oper. Res. (2021) 1-45. DOI: 10.1007/s10479-020-03871-7.

[21] S. Khalilpourazari and H.H. Doulabi, Robust modelling and prediction of the COVID-19 pandemic in Canada. Int. J. Prod. Res. (2021) 1-17. DOI: 10.1080/00207543.2021.1936261.

[22] S. Khalilpourazari and S.H.R. Pasandideh, Designing emergency flood evacuation plans using robust optimization and artificial intelligence. J. Comb. Optim. 41 (2021) 640-677.

[23] S. Khalilpourazari, H.H. Doulabi, A.O. Ciftcioglu and G.W. Weber, Gradient-based grey wolf optimizer with Gaussian walk: Application in modelling and prediction of the COVID-19 pandemic. Expert Syst. Appl. 177 (2021) 114920. DOI: $10.1016 / j$.eswa. 2021.114920 .

[24] S. Lalotra and S. Singh, Knowledge measure of hesitant fuzzy set and its application in multi-attribute decision-making. Comput. Appl. Math. 39 (2020) 1-31.

[25] M. Larbani, Solving bimatrix games with fuzzy payoffs by introducing nature as a third player. Fuzzy Sets Syst. 160 (2009) 657-666.

[26] M. Larbani, Non cooperative fuzzy games in normal form: A survey. Fuzzy Sets Syst. 160 (2009) 3184-3210.

[27] D. Liang and Z.S. Xu, The new extension of TOPSIS method for multiple criteria decision making with hesitant pythagorean fuzzy sets. Appl. Soft Comput. 60 (2017) 167-179.

[28] H. Liao and Z.S. Xu, Some new hybrid weighted aggregation operators under hesitant fuzzy multi-criteria decision making environment. J. Intell. Fuzzy Syst. 26 (2014) 1601-1617.

[29] R. Lotfi, M. Nayeri, S. Sajadifar and N. Mardani, Determination of start times and ordering plans for two-period projects with interdependent demand in project-oriented organizations: A case study on molding industry. J. Proj. Manag. 2 (2017) $119-142$.

[30] R. Lotfi, Z. Yadegari, S.H. Hosseini, A.H. Khameneh, E.B. Tirkolaee and G.W. Weber, A robust time-cost-quality-energyenvironment trade-off with resource-constrained in project management: A case study for a bridge construction project. $J$. Ind. Manag. Optim. 13 (2020) 1-22.

[31] R. Lotfi, N. Mardani and G.W. Weber, Robust bi-level programming for renewable energy location. Int. J. Energy Res. 45 (2021) 7521-7534.

[32] R. Lotfi, B. Kargar, S.H. Hoseini, S. Nazari, S. Safavi and G.W. Weber, Resilience and sustainable supply chain network design by considering renewable energy, Int. J. Energy Res. (2021) DOI: 10.1002/er.6943.

[33] J.M. Merigo, A unified model between the weighted average and induced OWA operator. Expert Syst. Appl. 38 (2011) 1156011572.

[34] X. Mo, H. Zhao and Z.S. Xu, Feature-based hesitant fuzzy aggregation method for satisfaction with life scale. Appl. Soft Comput. 94 (2020). DOI: 10.1016/j.asoc.2020.106493.

[35] P. Mula, S.K. Roy and D.F. Li, Birough programming approach for solving bi-matrix games with birough payoff elements. J. Intell. Fuzzy Syst. 29 (2015) 863-875.

[36] I. Nishizaki and M. Sakawa, Fuzzy and multiobjective games for conflict resolution, Physica-Verlag, Heidelberg (2001).

[37] T. Parthasarathy and T.E.S. Raghavan, Some topics in two-person games, American Elsevier Publishing Company, New York (1971).

[38] S.K. Roy, Game theory under MCDM and fuzzy set theory, VDM (Verlag Dr. Muller), Germany (2010).

[39] S.K. Roy and P. Mula, Bimatrix game in bifuzzy rough environment. J. Uncertainty Anal. Appl. 1 (2013) 11.

[40] S.K. Roy and P. Mula, Rough set approach to bimatrix game. Int. J. Oper. Res. 23 (2015) 229-244.

[41] S.K. Roy and S.N. Mondal, An approach to solve fuzzy interval valued matrix game. Int. J. Oper. Res. 26 (2016) $253-267$.

[42] S.K. Roy and P. Mula, Solving matrix game with rough payoffs using genetic algorithm. Oper. Res. Int. J. 16 (2016) 117-130.

[43] S.K. Roy and A. Bhaumik, Intelligent water management: a triangular type-2 intuitionistic fuzzy matrix games approach. Water Resour. Manag. 32 (2018) 949-968.

[44] S.K. Roy and J. Jana, The multi-objective linear production planning games in triangular hesitant fuzzy sets. Sadhana 46 (2021) 176.

[45] M. Sakawa and H. Yano, Interactive decision making for multiobjective non linear programming problems with fuzzy parameters. Fuzzy Sets Syst. 29 (1989) 315-326.

[46] M. Sakawa and I. Nishizaki, Max-min solution for fuzzy multiobjective matrix games. Fuzzy Sets Syst. 67 (1994) 53-69.

[47] S. Singh and S. Lalotra, On generalized correlation coefficients of the hesitant fuzzy sets with their application to clustering analysis. Comput. Appl. Math. 38 (2019) 1-26.

[48] G. Sun, X. Guan, X. Yi and Z. Zhou, An innovative TOPSIS approach based on hesitant fuzzy correlation coefficient and its applications. Appl. Soft Comput. 68 (2018) 249-267.

[49] V. Torra and Y Narukawa, On hesitant fuzzy sets and decision, In: Proceedings of the IEEE International Conference on Fuzzy Systems, Jeju Island, Korea (2009) 1378-1382.

[50] C.Y. Wang and S.M. Chen, Multiple attribute decision making based on interval-valued intuitionistic fuzzy sets, linear programming methodology, and the extended TOPSIS method. Inf. Sci. 397 (2017) 155-167.

[51] M.M Xia and Z.S. Xu, Hesitant fuzzy information aggregation in decision making. Int. J. Approx. Reason. 52 (2011) $395-407$.

[52] S.H. Xiong, Z.S. Chen and K.S. Chin, A novel MAGDM approach with proportional hesitant fuzzy sets. Int. J. Comput. Intell. Syst. 11 (2018) 256-271. 
[53] R.R. Yager, On ordered weighted averaging aggregation operators in multi-criteria decision making. IEEE Trans. Syst. Man Cybern. 18 (1988) 183-190.

[54] K.P. Yoon and W.K. Kim, The behavioral TOPSIS. Expert Syst. Appl. 89 (2017) 266-272.

[55] L.A. Zadeh, Fuzzy sets. Inf. Control. 8 (1965) 338-356.

\section{Subscribe to Open (S20) A fair and sustainable open access model}

This journal is currently published in open access under a Subscribe-to-Open model (S2O). S2O is a transformative model that aims to move subscription journals to open access. Open access is the free, immediate, online availability of research articles combined with the rights to use these articles fully in the digital environment. We are thankful to our subscribers and sponsors for making it possible to publish this journal in open access, free of charge for authors.

\section{Please help to maintain this journal in open access!}

Check that your library subscribes to the journal, or make a personal donation to the $\mathrm{S} 2 \mathrm{O}$ programme, by contacting subscribers@edpsciences.org

More information, including a list of sponsors and a financial transparency report, available at: https://www. edpsciences.org/en/maths-s2o-programme 